Article

\title{
Vallées glaciaires et fjords : réflexions à partir de l'exemple islandais
}

\author{
Jean-Noël Salomon ${ }^{凶}$ \\ ${ }^{1}$ Université Michel de Montaigne Bordeaux 3, France.
}

\begin{abstract}
Résumé. Les glaciations engendrent des glaciers, c'est une évidence. Mais ces derniers ne sont pas identiques, ils varient en fonction de leur masse, de leur position sur les reliefs (sommitale ou non), de la température de leur glace, de leurs écoulements et... du temps. Les actions glaciaires sont donc extrêmement variées et laissent des traces nombreuses, ce qui permet aux géomorphologues de reconstituer l'évolution des paysages où ils ont sévi. Parmi les traces on relèvera les fjords. Ces anciennes vallées glaciaires envahies par la remontée de la mer sont particulièrement spectaculaires du fait de leur jeunesse et des paysages qu'elles génèrent (hautes parois sub-verticales, cascades, épaulements, vallées suspendues, aspect sauvage, etc.) qui attirent les touristes et les croisiéristes. Les plus connus, véritables prototypes géomorphologiques sont les fjords norvégiens. Mais il existe bien d'autres pays à fjords (Groenland, Alaska, Patagonie chilienne, Ile Sud de Nouvelle-Zélande, etc.) et donc, par-delà leurs caractères communs, des différences notables existent. Parmi ces dernières, les fjords d'Islande offrent des caractéristiques inhabituelles : largeurs exceptionnelles, encaissement relativement peu profond (rareté des surcreusements) et surtout de nombreux dépôts sédimentaires à l'aval allant jusqu'à former des lidos. Le but de cet article est de récapituler les connaissances en matière de géomorphologie glaciaire et de montrer le particularisme des fjords islandais. Enfin, des hypothèses explicatives de ce dernier sont avancées, appuyées par des photographies.

Mots clés : Glaciers, actions glaciaires, vallées glaciaires, types de fjords, Islande.
\end{abstract}

CORRESPONDANCE :

$\triangle$ Institut de Géographie Louis Papy, Université Michel de Montaigne-Bordeaux 3; Campus universitaire de PESSAC (33 607).
HISTOIRE DE L'ARTICLE :

Reçu : 15 Septembre 2018

Accepté : 17 Septembre 2018

Disponible en ligne : 10 Octobre 2018

Мjnsalomon@yahoo.com 


\section{Introduction}

Les vallées glaciaires, dont parfois les parties aval ont été envahies par la mer lors de la remontée flandrienne (fjords), ont été modelées principalement par l'action des glaciers. Il existe une énorme littérature sur le sujet, accessible notamment au travers des manuels de géomorphologie et Internet, si bien que nous n'y ferons pas référence bien que de nombreux constats expliqués ci-dessous y sont explicités. En effet, le texte qui suit se nourrit principalement de nos observations de voyage effectuées soit en haute montagne (Europe et Amériques), soit dans des pays de hautes latitudes (Spitzberg, Scandinavie, Ecosse, Islande pour l'hémisphère nord ; Andes chiliennes et argentines de Patagonie, Nouvelle-Zélande, pour l'hémisphère sud). Les nombreuses illustrations qui accompagnent et appuient le texte en sont issues. Toutefois il existe de bons manuels et l'on pourra consulter avec intérêt celui, ancien, de Martonne E. (1910), les manuels classiques de géomorphologie (ex : Derruau M. (1965), ou plus spécialisé et récent de Zryd (2001), ou articles (Ahlmann H.W., 1954 ; Bout et al., 1955) sans compter les innombrables sites Internet (inégaux).

Comme attendu, points communs (processus) et différences (morphologies) apparaissent. La discussion porte sur différentes genèses et les hypothèses explicatives des paysages qui en résultent.

\section{La genèse des glaciers et paramètres explicatifs de l'aspect des vallées glaciaires}

Les données tectoniques (failles et fractures jouant sur leur orientation) sont à prendre en compte d'emblée car ce sont elles qui déterminent l'essentiel des orientations générales ou locales prises par les glaciers qui ont emprunté les paléovallées d'avant les glaciations. Les retouches éventuelles sont liées aux débordements en cas de forte alimentation, mais l'essentiel des axes reste inscrit dans les paysages. Ensuite, il faut tenir compte du rôle du froid quant à la formation des glaciers. Une altitude minimale ou une latitude élevée sont nécessaires pour l'obtention des conditions requises à la formation d'un glacier car seule une température proche de $0^{\circ} \mathrm{C}$ permet une précipitation neigeuse et non pluviale. Enfin, le facteur temps pose la question de la durée nécessaire au glacier pour que celui-ci façonne la vallée qu'il parcourt mais aussi celle des héritages glaciaires car, même si le glacier a reculé ou disparu (cas très fréquent), les modelés glaciaires subsistent, très reconnaissables (formes creusées dans les roches ou dépôts variés).

Par ailleurs, on sait que la formation d'un glacier résulte d'un bilan, dit bilan de masse. Au départ, il faut une précipitation neigeuse et que les flocons s'accumulent. Peu à peu, la neige, écrasée sous son propre poids, expulse l'air qu'elle contient. De plus, le jour, elle peut fondre légèrement en surface sous l'action de la chaleur solaire, mais la nuit les températures s'abaissent et l'eau congèle pour se 
transformer en glace. Le processus se répétant, la glace initialement bulleuse devient de plus en plus compacte (et plus bleue). Il existe des sites plus propices que d'autres à l'accumulation (topographie, exposition au vent, etc. Un glacier peut alors se former dans des cirques de morphologies variées (en fauteuil, en van, en pelle, etc.). Comme la masse glaciaire est susceptible de fluer, une langue glaciaire peut sortir $\mathrm{du}$ site et parcourir la vallée en contrebas. Dans le cas des calottes glaciaires il s'agit de glaces «froides" à élaboration beaucoup plus lente, mais le fluage se produit également.

Ce faisant la glace s'abaisse en altitude et donc les températures croissantes finissent par amener une fonte, notamment en saison estivale. Les pluies éventuelles aident souvent à la fonte dans la mesure où l'eau est plus chaude que la glace et qu'elle circule (frottements). Il en est de même des poussières ou cendres volcaniques (de couleur sombre), voire de certains blocs de roche qui saupoudrent ou jonchent parfois les surfaces. En effet, les éléments noirs accumulent plus de chaleur, ce qui accentue la fonte autour d'eux : beaucoup s'enfoncent verticalement dans le glacier. Deux autres processus entrent également en jeu : le glissement de la masse d'eau gelée vers l'aval et la sublimation (passage direct de l'eau de l'état solide à l'état gazeux). Longtemps sous-estimé ce dernier processus est pourtant très important notamment en Antarctique ou encore dans les hautes montagnes où par exemple il explique la formation des pénitents (aiguilles de glaces légèrement obliques, orientées selon le soleil).

Pour que le glacier se maintienne, il faut donc que l'apport en neige excède (ou équilibre) la perte de glace. La compréhension de ce bilan est fondamentale. Certains glaciers sont remarquablement alimentés. C'est le cas du Mont Cook (3 724 $\mathrm{m}$ et plus de $8000 \mathrm{~mm}$ de précipitations neigeuses) dans les Alpes de NouvelleZélande, pourtant il est en recul (200 m en 20 ans) car la température moyenne en aval a légèrement augmenté. C'est aussi le cas de tous les glaciers de Patagonie, très bien alimentés $(\mathrm{P}>7000 \mathrm{~mm})$ mais tous en recul, ou encore des Alpes norvégiennes (sommets > à $2000 \mathrm{~m}$ et $\mathrm{P}>$ à $2000 \mathrm{~mm}$ ).

Le bilan doit être annuel car le phénomène saisonnier joue. En période estivale, les précipitations nivales cèdent souvent la place à des pluies ce qui accélère la fonte. Le bilan est alors négatif. Au contraire, avec les mois les plus froids, les précipitations neigeuses l'emportent, les stocks de glace se reconstituent et le bilan redevient positif.

Un cas spécial est celui des glaciers tropicaux (Popocatépetl, Kilimandjaro, Mt Karstens, Andes péruviennes, etc.) qui sont surtout alimentés en saison chaude car c'est la saison des pluies, et donc... celle des neiges.

\section{L'action glaciaire}

Sous l'effet de son poids, de la gravité et de la pente un glacier s'écoule lentement par fluage car lorsqu'il est assez épais il acquiert une certaine plasticité (il 
peut donc se déformer). D'une façon générale, plus la masse est importante, plus la pente est forte, et plus la température est "élevée », plus le glacier est susceptible $\mathrm{d}^{\prime}$ avancer. De façon pragmatique, on distingue une "glace froide " $\left(-6^{\circ} \mathrm{C},-7^{\circ} \mathrm{C}\right.$ et jusqu'à $-20^{\circ} \mathrm{C}$ et moins) et une "glace chaude $\left(-1^{\circ} \mathrm{C},-2{ }^{\circ} \mathrm{C}\right.$, voire $\left.-3^{\circ} \mathrm{C}\right)$. En effet, avec une température " chaude», le glacier contient plus d'eau liquide et de l'air, ce qui favorise la fonte (torrents sous-glaciaires, poches d'eau, semelles glaciaires, etc.) et, à la base, l'avancée par glissement.

Cette dernière provoque une action de creusement de la vallée empruntée par frottement, abrasion, arrachement, délogement, etc., surtout si le glacier est armé de moraines (blocs éboulés des parois et amassés dans un premier temps en surface, puis redistribués à mesure de l'avancement d'où la distinction entre les différentes formes de moraines: latérales, médianes, de fond, etc.) et de sédiments broyés jouant un rôle d'abrasif. Les formes de cette abrasion sont bien connues : microformes: polis, stries, cannelures, croissants, cannelures ; ou roches moutonnées) (Photos 1 et 2) L'eau de fonte peut aussi agir directement par frottement voire par dissolution (si l'encaissant est karstifiable).

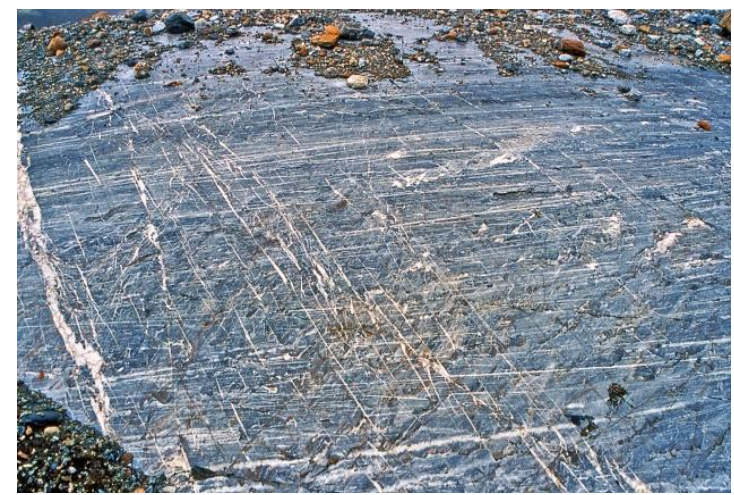

Photo 1 : Stries glaciaires particulièrement visibles car elles recoupent perpendiculairement de petits filons de calcite ce qui donne un quadrillage. Les stries indiquent le sens de l'écoulement du glacier. (Spitzberg). (cliché. J-N Salomon).

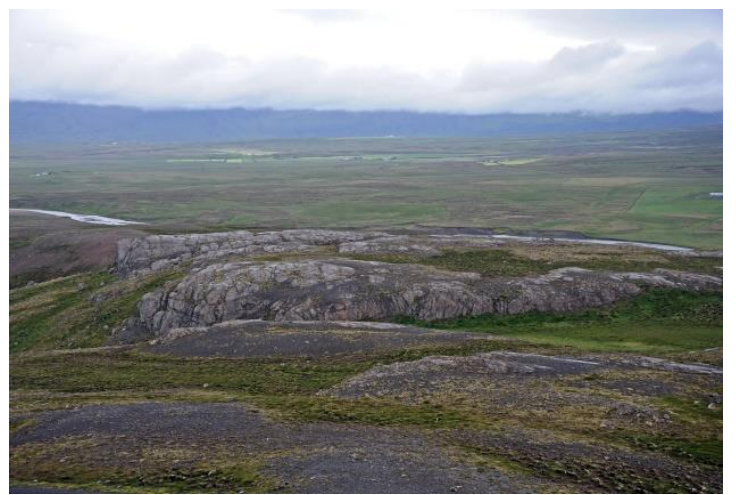

Photo 2 : Vallée glaciaire de la Vididalsa (Nord islandais) particulièrement large. Les roches moutonnées sont incisées par des stries et des cannelures et les torrents sous-glaciaires ont creusé des gorges d'incision sur verrou. (cliché. J-N Salomon).

Localement, en fonction de la topographie, la surface est couverte de crevasses (au-dessus des seuils rocheux et verrous) ou de séracs (cuvettes) car la glace casse (Photo 3). A plus grande échelle, il peut se produire des surcreusements (ultérieurement remplis par des lacs) ou de gros dépôts (moraines frontales) plus ou moins redistribués par les eaux fluvio-glaciaires et les crues brutales.

En Islande, la glaciation a laissé des traces partout. Chaque avancée et chaque recul a été enregistré dans la vallée glaciaire sous forme de creusements liés à l'abrasion et bien reconnaissables (stries, cannelures, polis, broutures, roches moutonnées, débitages, etc.) ; ou de dépôts (moraines, sédiments broyés, blocs erratiques, tillites, 
sandurs comme entre Fagurhólsmýri et Kirkjubæjarklaustur, dans le Sud). Et l'eau de fonte (torrents sous-glaciaires ou de bordure) a engendré de profondes incisions (gorges) notamment sur les verrous.

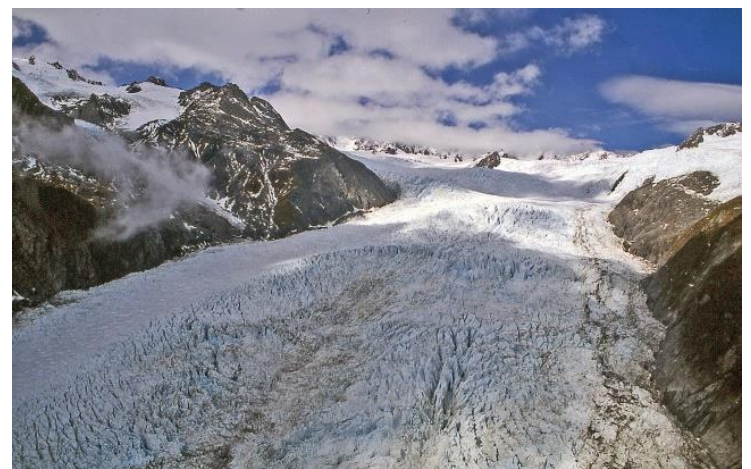

Photo 3 : La surface du glacier François-Joseph (NouvelleZélande). La glace de ce glacier de $12 \mathrm{~km}$ progresse en moyenne de $250 \mathrm{~m} / \mathrm{an}$ car les précipitations neigeuses sont très abondantes, ce qui n'empêche pas le front du glacier de reculer depuis 2008. S'il descend encore jusqu'à 300 m d'altitude, il n'atteint plus la mer par manque d'alimentation conséquente et d'importantes fontes. Il n'a pas eu le temps de former un fjord. En amont, la glace progresse sur des fonds rocheux inégaux ce qui se traduit par une multiplication de séracs et de crevasses (cliché. JN Salomon).

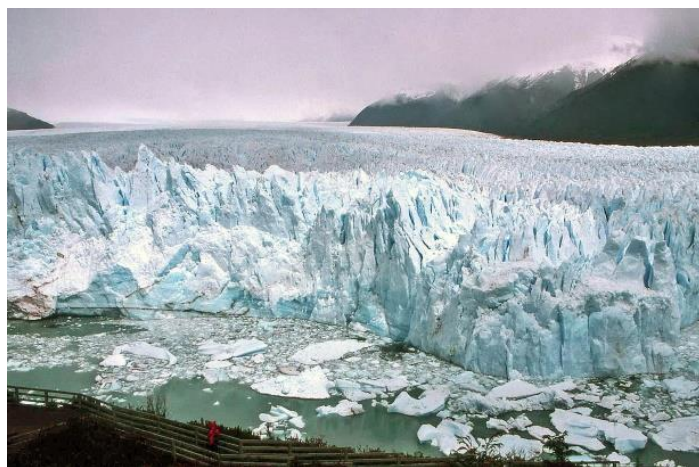

Photo 4 : Front glaciaire du Perito Moreno (Patagonie argentine) et vêlage régulier de « tempanos » (icebergs lacustres). Le front mesure 5 $\mathrm{km}$ de large et s'élève de $80 \mathrm{~m}$ au-dessus du niveau du lac.

Des vallées affluentes en « $U$ » sont dites suspendues lorsque la connexion avec le glacier principal ne se fait plus. Le réseau de vallées glaciaires interconnectées est alors rompu.

Pendant longtemps les géomorphologues ont cherché à savoir quelles étaient les avancées maximales des glaciers (et des grandes glaciations), mais aussi des pulsations glaciaires. Pour cela on regardait où se situaient les moraines terminales, ou encore on établissait des repères (parfois on jalonnait le glacier de piquets). Mais ces estimations étaient sommaires et souvent erronées. Par exemple, on a souvent confondu l'avancée de la glace (toujours réelle) avec celle du glacier (en fait le front du glacier). Par exemple le Perito Moreno, en Argentine, progresse de 5m/jour en moyenne, mais son front reste stable, voire recule (Photo. 4). Pourtant bien des auteurs citent ce glacier comme étant en progression, oubliant que ses voisins (Spegazzini et Upsala au nord; Grey au sud), soumis aux mêmes conditions climatiques, sont en recul. Et certaines progressions du front traduisent en fait davantage un amaigrissement $\mathrm{du}$ glacier qu'une croissance. Ces crues glaciaires temporaires (surges) s'expliquent par le réchauffement de la glace (cf. supra) qui favorise le glissement. En fait une appréciation sérieuse doit se faire, comme nous avions procédé au Spitzberg (mission franco-polonaise et navire océanographique 
l’Horyzont II - 2011), avec des mesures radar pour apprécier le profil transversal de la vallée et quantifier le volume de glace (gagné ou perdu). L'observation des parois donne également de précieuses indications (Photo. 5). En fait, tous les glaciers du Spitzberg sont en recul depuis plus de 60 ans.

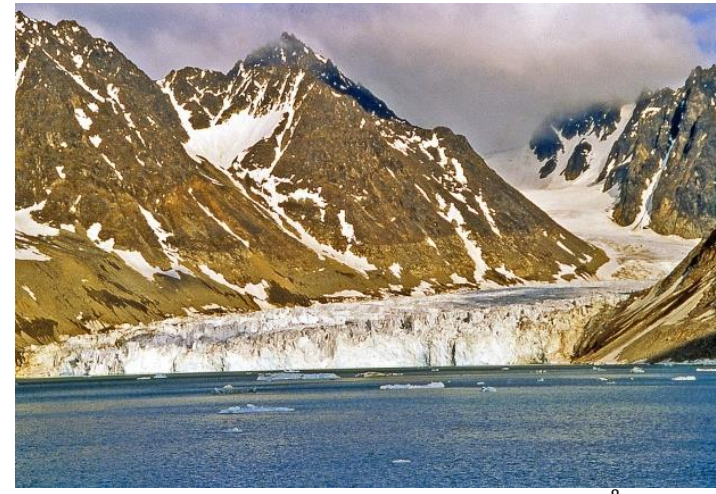

Photo 5 : Surge ou crue glaciaire près de Ny Ålesund (Spitzberg). La crue glaciaire se traduit par une avancée rapide de la glace, mais temporaire. Il s'ensuit un recul du front glaciaire et une diminution du volume de celui-ci. On observe très bien cet amaigrissement sur les parois : couleur plus claire sur environ $25 \mathrm{~m}$ laquelle se prolonge au-delà de l'avancée maximale du glacier actuel.

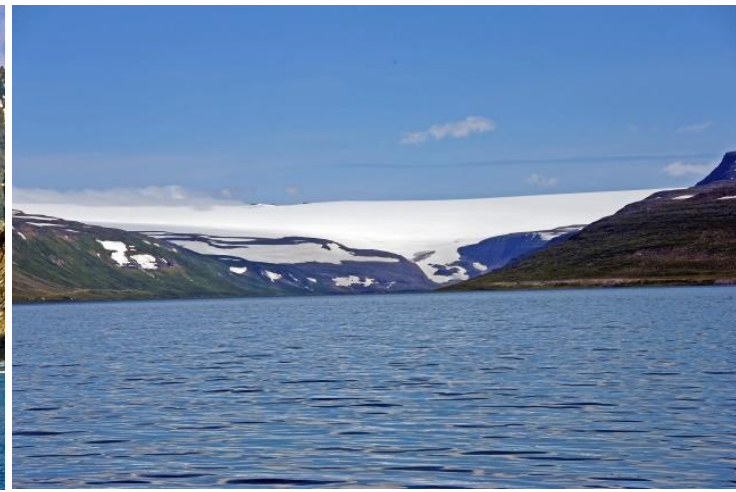

Photo 6 : Calotte du Drangajokull. Cette calotte de moins de $400 \mathrm{~km} 2$ envoie encore des émissaires vers la mer, mais ils n'y parviennent plus. Ils sont cependant à l'origine du fjord d'Isafjardardjup et de superbes vallées suspendues (cliché. J-N Salomon).

\section{Aperçu des grands types de glaciers}

$\mathrm{D}^{\prime}$ une façon classique on peut distinguer les glaciers non contraints par la topographie (inlandsis - calottes glaciaires et glaciers émissaires), de ceux qui le sont (glaciers de cirque, de vallées, de transsection et de piémont).

Les inlandsis (mot à mot "glace de l'intérieur des terres ») sont définis arbitrairement par leur étendue $\left(>50000 \mathrm{~km}^{2}\right)$ et au fait qu'ils recouvrent entièrement les terrains qu'ils occupent du fait de leur très grande épaisseur de glace. Il existe sous la glace des reliefs escarpés, formant parfois des montagnes et de rares sommets percent la glace : les nunataks.

De nos jours ils ne sont plus que deux: l'Antarctique et le Groenland. L'inlandsis de l'Antarctique couvre 15 millions de $\mathrm{km}^{2}$ et la couche de glace (environ 28 millions de $\mathrm{km}^{3}$ ) peut dépasser, par endroits, 4,7 km d'épaisseur. Les dimensions de l'inlandsis du Groenland sont également très importantes : $1700000 \mathrm{~km}^{2}$ et la glace peut atteindre l'épaisseur de $3000 \mathrm{~mm}$ au centre. Le volume global de glace est estimé à $2000000 \mathrm{~km}^{3}$ de glace. Mais au Pléistocène il en existait bien d'autres : l'inlandsis laurentidien (Canada), celui de Patagonie, l'islandais (Van Viet Lanoe, 2010), le scandinave et plusieurs dans les hautes montagnes (Himalaya, Altaï, Monts Verkhoïansk, etc.). Ils ont fondu et laissé des traces telles les Urstromtälers (« vallées 
primitives ») générées par les eaux de fonte sur le front de l'inlandsis scandinave en retrait et qui rejoignaient la mer du Nord. Aujourd'hui, la grande plaine allemande est criblée de nombreux lacs, parsemée de monticules morainiques postglaciaires et imprimée de larges vallées (servant d'axes de circulation au réseau routier) générées il y a quelques 13000 à 16000 ans.

Les calottes glaciaires (ice caps) sont plus petites mais recouvrent encore entièrement leur soubassement montagneux. Cependant, en raison des pentes la glace peut s'écouler sur les bordures en générant des lobes glaciaires, des courants glaciaires avec parfois des digitations. Les plus grandes calotte glaciaires actuelles sont celles d'Agassiz de l'île d'Ellesmere, au Canada $\left(21000 \mathrm{~km}^{2}\right)$, de Severny $\left(20500 \mathrm{~km}^{2}\right)$ en Nouvelle-Zemble, des iles canadiennes Axel Heiberg, Devon et de Baffin (environ $\left.19000 \mathrm{~km}^{2}\right)$ des Hielos patagónicos $\left(16.800 \mathrm{~km}^{2}\right)$ en Patagonie et du Spitzberg $\left(8400 \mathrm{~km}^{2}\right)$.

La calotte du Vatnajökull (Islande) vient bien après, avec $8100 \mathrm{~km}^{2}$. Au sud, elle est bien alimentée en neige $(P>2300 \mathrm{~mm}$ ), fournit des glaciers émissaires qui viennent jusqu'à la mer (Ahlmann et Thorarinsons, 1938). Mais au nord, moins fournie, la glace émissaire descend rarement en deçà des $1000 \mathrm{~m}$. L'Islande possède d'autres calottes : le Langjökull (<1 $400 \mathrm{~m}$ et $\left.1000 \mathrm{~km}^{2}\right)$, le Hofjökull (1 $763 \mathrm{~m}$ et 950 $\mathrm{km}^{2}$ ), le Myrdallsjökull (1 $450 \mathrm{~m}$ et $680 \mathrm{~km}^{2}$ ), et le Drangajökull le plus petit (925 m et $<400 \mathrm{~km}^{2}$ ) (Photo. 6)

D'une façon générale, les calottes glaciaires qui ont souvent mis plusieurs centaines de milliers d'années pour se constituer et dépassent parfois les $1000 \mathrm{~m}$ d'épaisseur, sont logiquement plus épaisses au centre. Elles s'écoulent donc vers l'extérieur à partir d'un dôme central. Ce faisant, la glace d'un glacier émissaire érode la semelle rocheuse en "emprisonnant " l'écoulement entre ses parois et, avec le temps, creuse une gorge. Ainsi, selon la topographie, toute une série de glaciers émissaires évacuent la glace du dôme vers l'extérieur. Le Vatnajökull illustre à merveille ce processus.

Les glaciers de vallée ou langues glaciaires, ou encore glaciers de type alpin, sont le prototype de la représentation classique que l'on se fait d'un glacier. Ces langues sont l'émanation de glaces accumulées dans un bassin d'alimentation en amphithéâtre, d'où le nom de cirque qu'on lui donne (Photo. 7) et qui fluent vers la vallée. Dans les Alpes d'excellents exemples sont ceux du glacier d'Aletsch (Suisse) ou de la Mer de Glace (France). Concentrée entre les parois, la langue glaciaire descend vers l'aval tout en recevant des glaciers affluents adjacents (parfois suspendus) et des éboulis issus des versants ce qui forme des moraines latérales (amas qui avancent portés par la glace). A mesure de son avancée, l'altitude baissant, la glace a tendance à fondre ce qui génère des écoulements de surface, intraglaciaires (avec parfois des poches d'eau) et sous-glaciaires. Finalement l'avancée du 
glacier cesse. Il dépose alors ses moraines (moraines frontales) sur son front (Photo. 8). Plusieurs avancées ou reculs successifs peuvent donner des cordons successifs. Un torrent (ou plusieurs) fluvio-glaciaire sort généralement du front et celui-ci étale, lors des crues, des sédiments et des moraines ce qui construit un cône fluvio-glaciaire (Photo. 9). Au cours de son avancée qui peut atteindre plusieurs centaines de kilomètres, la langue glaciaire modèle fortement la vallée en lui donnant une forme en « $\mathrm{U}$ » caractéristique. Mais dans bien des cas, ce profil «idyllique » ne se retrouve pas car les actions ultérieures d'érosion ont retouché ce profil type. Une variante est le glacier rocheux. Souvent employé à tort car mal compris, le terme de glacier rocheux doit être utilisé pour décrire un corps de glace recouvert de débris produits selon différents processus. Les glaciers rocheux se caractérisent par une avancée produisant des rides arquées. Par essence, ils concernent essentiellement les régions périglaciaires, particulièrement aptes à produire des débris venant recouvrir le glacier.

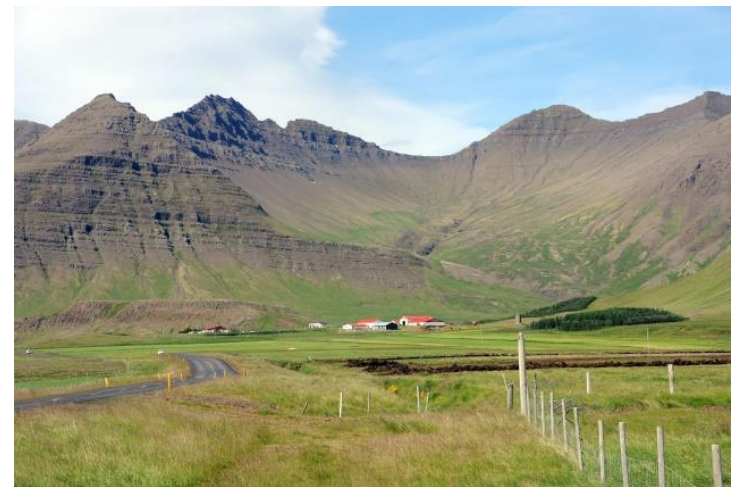

Photo 7 : Cirque glaciaire à Gerdubert (W

Islande). Aujourd'hui il n'abrite plus que des névés en hiver, mais il fut le point de départ d'un glacier allant jusqu'à la mer. (cliché. J-N Salomon).

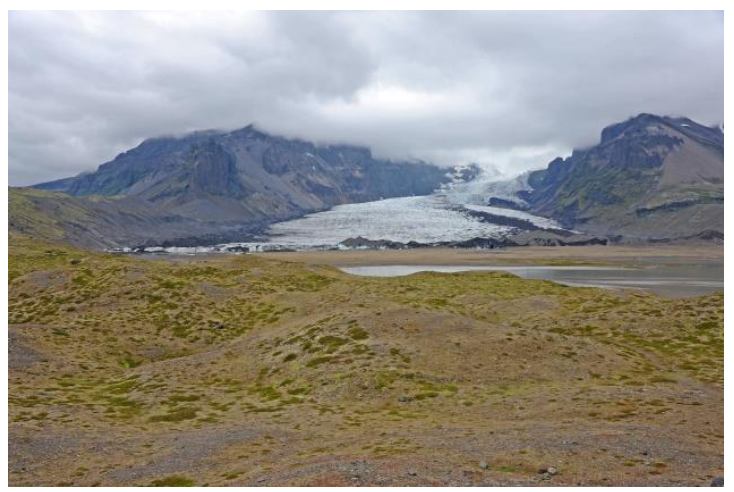

Photo 8 : Langue de retrait du Fjallsjokull, glacier émissaire du Vatnajökull. Moraine frontale, latérales et lac de front glaciaire. Photographie prise depuis la moraine frontale faisant barrage. (cliché. J-N Salomon).

Le glacier de piémont est une variante : il s'agit d'un glacier qui atteint la plaine où l'encaissement n'est plus guère possible. Dès lors sa zone d'ablation s'étale soit en digitations soit en lobe. Se forme alors un sandur (Photo. 10) où se multiplient des formes spécifiques : moraines, drumlins, eskers et kames, kettles, et abandons de blocs erratiques. Le plus grand glacier de piémont est le Malaspina en Alaska (3900 km² et $38 \mathrm{~km}$ ). Le Vatnajökull, en Islande, en possède plusieurs.

$\mathrm{Au}$ final, il est toujours possible de proposer de nombreuses autres classifications en fonction des critères (étendues, longueurs, contraintes, genèses, etc.) que l'on retient (glacier suspendus, régénérés (Photo. 11), etc.). Les observations de terrain montrent que la réalité aboutit le plus souvent à des formes composites. De même il existe un grand nombre de petits glaciers à travers le monde qui 
s'apparentent plus à de gros névés compactés qu'à de vrais glaciers. Ils n'ont pas de grande action érosive même si certains peuvent générer des moraines de névé.

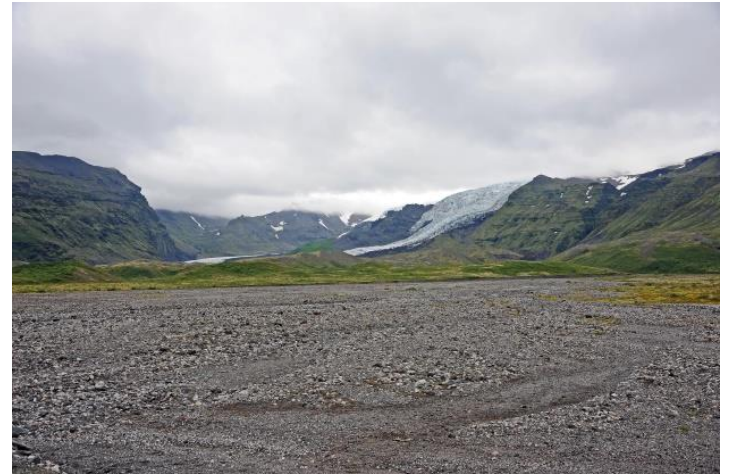

Photo 9 : Cône fluvio-glaciaire du Kviarjökull, émissaire du Vatnajökull. (cliché. J-N Salomon).

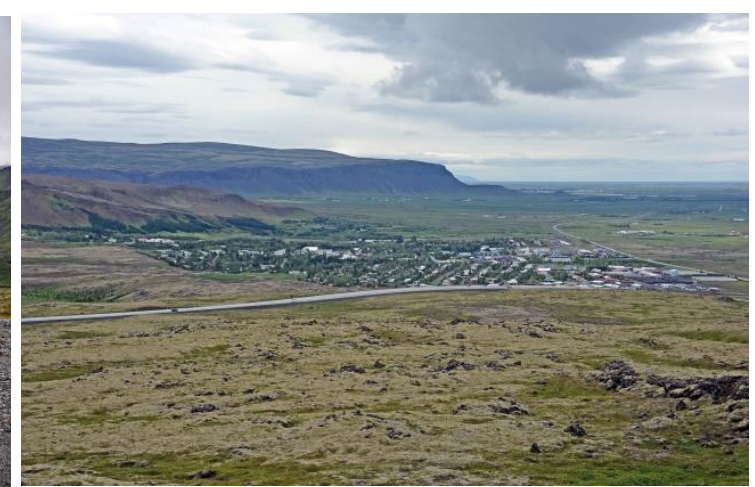

Photo 10 : Le Sudurland, vaste plaine fluvio-glaciaire de type sandur au sud du Vatnajökull. Elle a été construite en partie grâce aux jökulhlaups. (cliché. J-N Salomon).

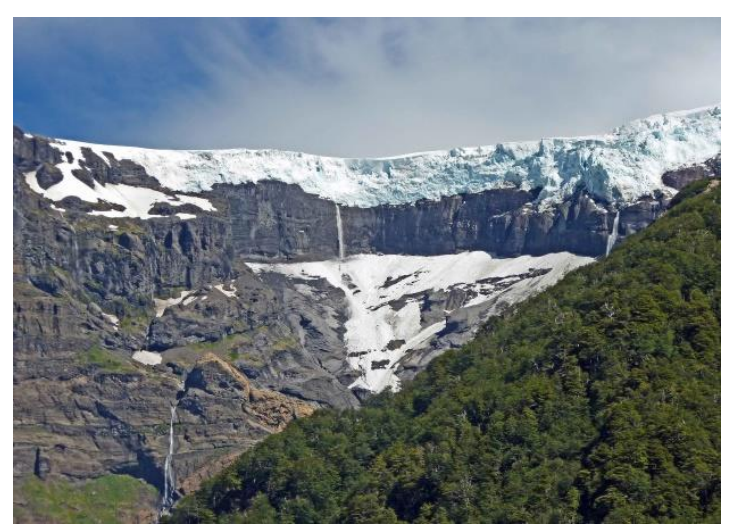

Photo 11 : Glacier suspendu du Cerro Tronador (3491 m) en Patagonie argentine. Son nom lui a été donné suite aux bruits de chutes régulières de pans de glacier (environ $200 \mathrm{~m}$ de haut) et produisant un bruit de tonnerre. Les blocs tombés se soudent par la suite et une langue glaciaire inférieure se reconstitue (non visible ici sur la photo). (cliché. J-N Salomon).

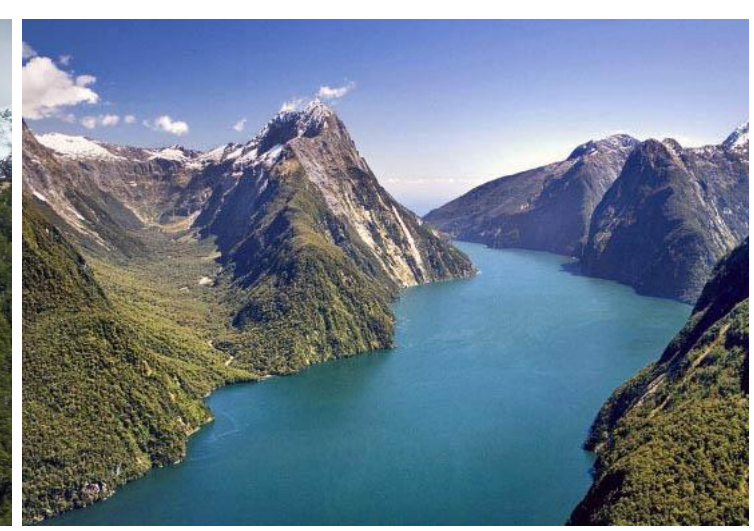

Photo 12 : Le Milford Sound. Situé dans l'Ouest de lîle Sud de Nouvelle-Zélande, ses parois encadrantes sont particulièrement élevées (jusqu'à plus de $1200 \mathrm{~m}$ ). Les chutes d'eau

(Shuterland, $580 \mathrm{~m}$; Stirling) y sont spectaculaires. Sa profondeur maximale atteint environ $400 \mathrm{~m}$. (cliché. J-N Salomon).

\section{Le cas de fjords islandais}

Rappelons qu'un fjord (terme norvégien) est une auge glaciaire occupée par la mer après la fonte du glacier et comme nous l'avons précisé dans un ouvrage précédant (SALOMON, 2008) les côtes à fjords s'étendent sur plus de $30000 \mathrm{~km}$. Les régions favorables sont les zones à latitudes élevés, les bordures soulevées des continents et celles des hautes chaînes ou volcans proches de la mer. Ce dernier cas concerne l'Islande. Il existe de nombreux termes pour nommer un fjord selon les régions 
(«firth», «loch» (Ecosse), «canales » (Patagonie chilienne); « sounds », « inlets » (Alaska, Groenland, Nouvelle-Zélande) (Photo. 12).

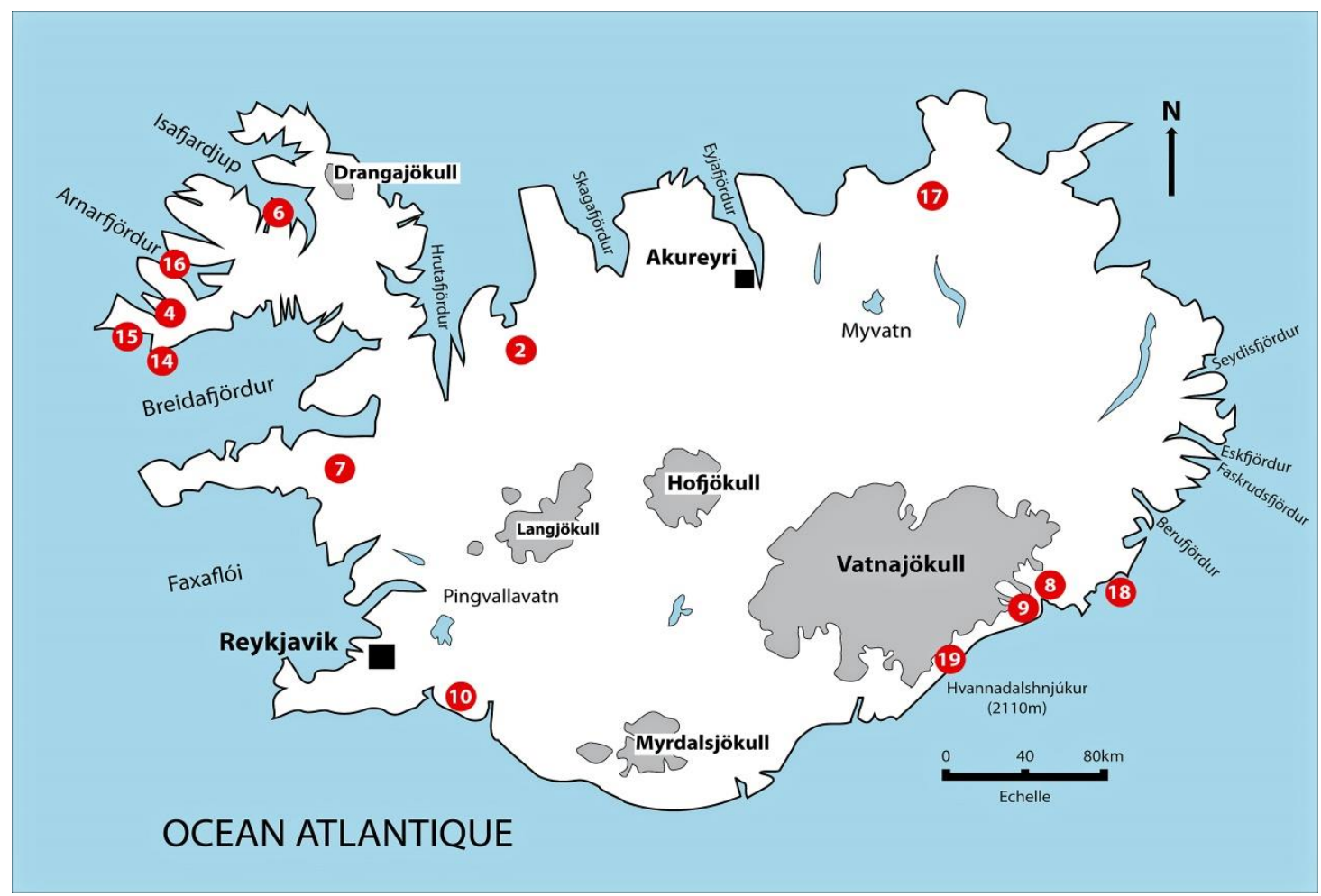

Figure 1 - Carte d'Islande avec les calottes glaciaires qui subsistent aujourd'hui, les principaux fjords et les sites de prise de vue des photographies

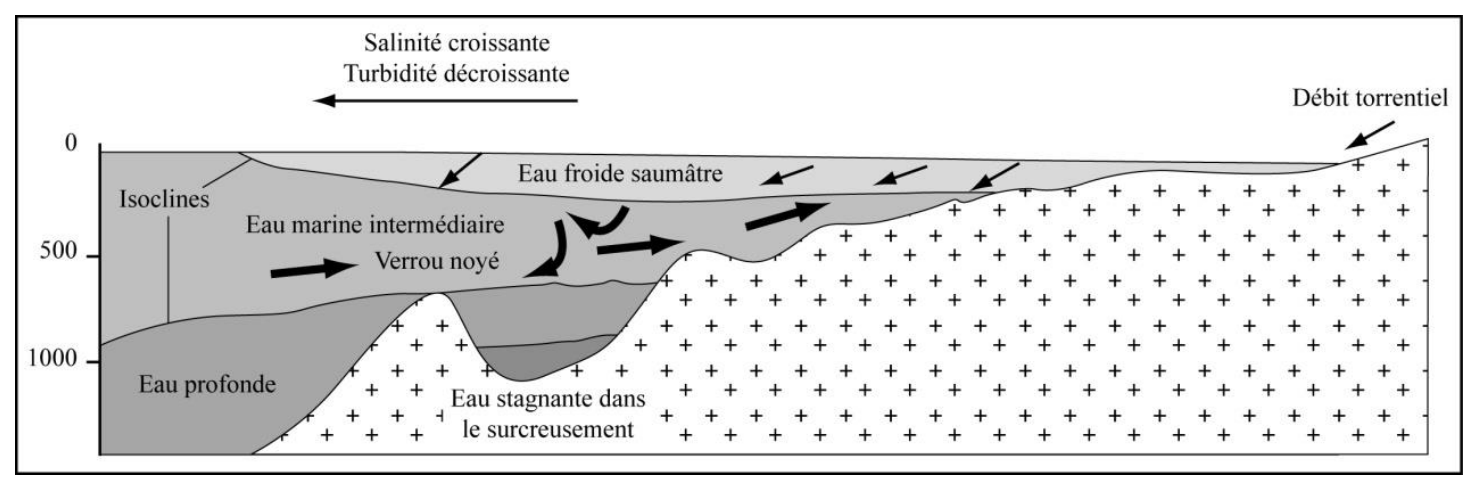

Figure 2 : Profil en long d'un fjord norvégien. On notera le surcreusement d'aval et le verrou noyé, caractéristique des fjords norvégiens liés à l'action du glacier, mais que l'on ne retrouve pas pour les fjords islandais, beaucoup plus larges.

Le prototyppe est le fjord norvégien qui est marqué par une forme en « $U$ ", une grande longueur (souvent plusieurs dizaines voire centaines de kilomètres), une largeur modeste en comparaison (de quelques centaines de mètres à $5-6 \mathrm{~km}$ ) et sur les côtés des vallées suspendues également en « $U$ », La profondeur est grande: 
jusqu'à $1308 \mathrm{~m}$ pour le Sognefjord, en Norvège (Photo. 13). Du fait d'un environnement montagneux jouxtant la mer (le Jotunheimen $(2469 \mathrm{~m})$ se trouve à moins de $50 \mathrm{~km}$ du Sognefjord), l'encaissement est remarquable avec de grandes vallées en auge dont seul l'amont émerge. Cela donne des parois très raides, parfois de plus de $1000 \mathrm{~m}$, agrémentées de replats multiples, étagés où s'accrochent à l'occasion des dépôts de plage anciennes et d'où descendent de magnifiques cascades. La mer cache en grande partie la valeur réelle de l'encaissement car il y a eu des surcreusements dus à l'érosion par les glaciers. Le surcreusement est particulièrement net à l'aval en raison de la pression du glacier sur le fond, mais comme la fonte s'exerce, la pression diminue ensuite fortement ce qui produit un seuil en aval du surcreusement (Figure 2). Par la suite, ces dépressions ont souvent été en partie colmatées car elles constituent des pièges à sédiments. Ceux-ci-sont plus abondants depuis le recul des glaciers (Figure 3).

En ce qui concerne les fjords islandais ce schéma ne correspond plus. En effet, ces derniers sont remarquablement larges (souvent plus de $10 \mathrm{~km}$ : Isafjordur, Arnarfjordur; et jusqu'à $25 \mathrm{~km}$ pour le l'Eyjafjordur; et même $50 \mathrm{~km}$ pour le Breidafjordur avec une superficie de $\left.2874 \mathrm{~km}^{2}\right)$ ! De plus ces fjords sont peu profonds : $168 \mathrm{~m}$ pour l'Isafjordur, $50 \mathrm{~m}$ Anarfjordur mais avec les hauts - fonds sableux de Langanes, de 20 à $65 \mathrm{~m}$ pour l'Eyjafjordur, $10 \mathrm{~m}$ pour le Skagafojordur et le Patreksfjordur et moins de $10 \mathrm{~m}$ pour le Reydarfjordur. D'où la construction fréquente de ponts (Borgrfjordur Eyjafjordur, Gilsfjordur, Kolgrafafjordur, etc.), voire de tunnels (Foraging- Hvalfjordur) pour traverser.

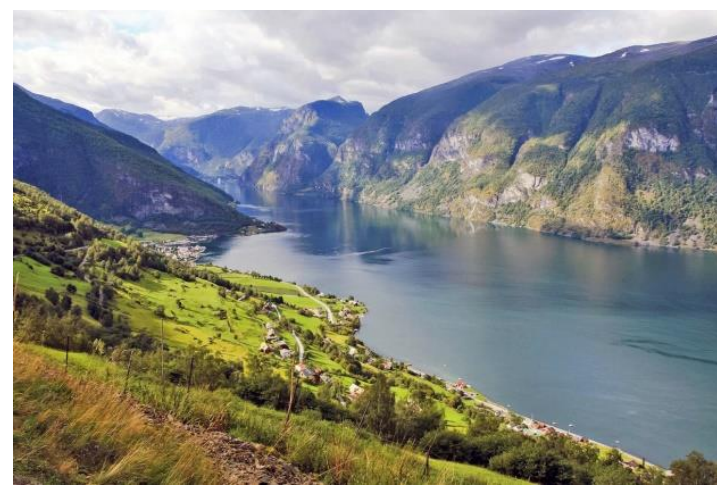

Photo 13 : Le Sognefjord. Situé au nord de Bergen

(Norvège), ce fjord est très grand (204 km) et très profond ( $1308 \mathrm{~m}$ en dessous du niveau de la mer) en raison du surcreusement lié à l'action glaciaire. Sa largeur moyenne est d'environ 4,5 $\mathrm{km}$ et les parois qui le surplombent, d'où cascadent de superbes chutes d'eau, dépassent parfois les $1000 \mathrm{~m}$. Le Sognefjord reçoit plusieurs branches affluentes dont le Naerofjord (20 km) est l'un des plus beau.

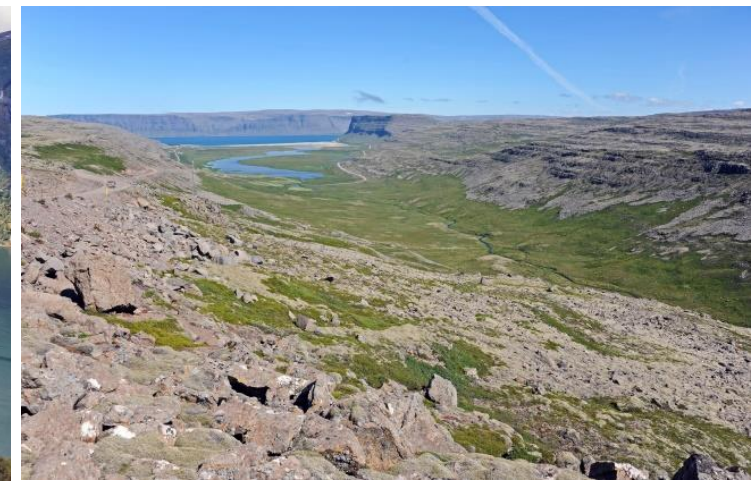

Photo 14 : Débouché du glacier du Storavahn qui mesurait $4 \mathrm{~km}$ de large à l'arrivée en mer.

Aujourd'hui, l'étalement des alluvions a généré le développement d'une plage sableuse qui obstrue le débouché sur le Patreksfjördur. (cliché. J-N Salomon). 


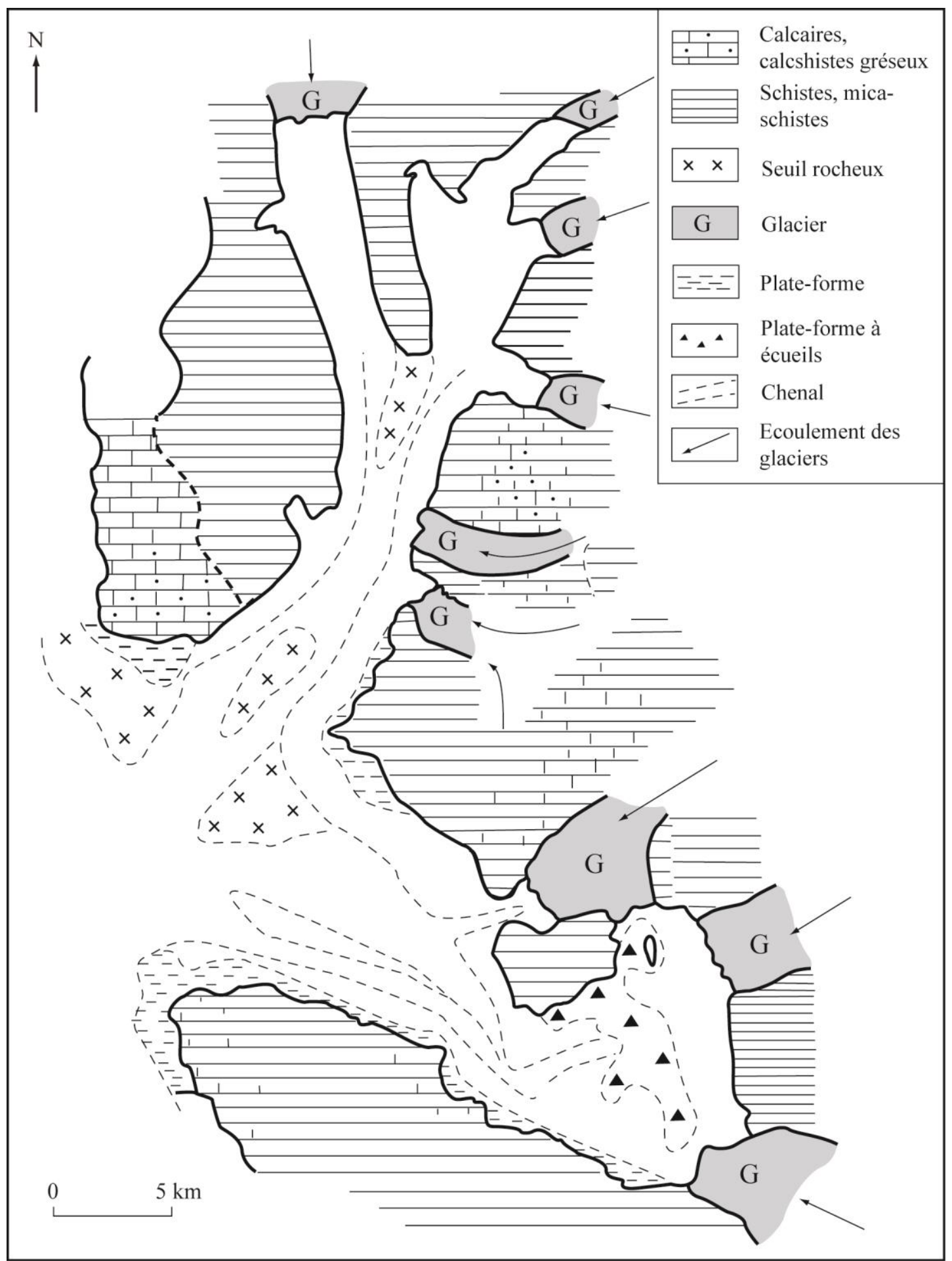

Figure 3 : Le Kongsfjord (Spitzberg), près de Ny-Âlesund, mesure 26 km de long et sa largeur varie de 6 à 14 $\mathrm{km}$. Il se subdivise aujourd'hui en deux branches : Kronebreen et Kongsvegen dont les deux têtes sont alimentées par de petits glaciers ce qui illustre un recul rapide des glaces. 
Cette faible profondeur a favorisé la sédimentation fluvio-glaciaire, d'autant que le climat actuel tend à empâter les thalwegs glaciaires (Photo. 14) par amas alluvionnaires grossiers, liés aux crues brutales, et même la remontée de sédiments redistribuée par les actions marines (nombreux lidos (Photo. 15), parfois isolant des lagunes (Hop; Vopnafjördur); tombolo double de Thordarhöfdi, au nord de Hofsos ; marais, voire petits deltas ; ou même actions éoliennes (dunes) (Photo.16). Au NE, au nord d'Egilstadir, l'ancien fjord s'est même transformé en lac simplement relié à la mer par un cours d'eau méandriforme qui a du mal à percer un lido de 16 $\mathrm{km}$ de long. La vallée glaciaire aboutissant à Breiddalsvik est également obturée par un lido générant une lagune dans laquelle la rivière Breidalsa a développé un delta. On retrouve des cas similaires dans le Sud-Est (Bodere, 1985).

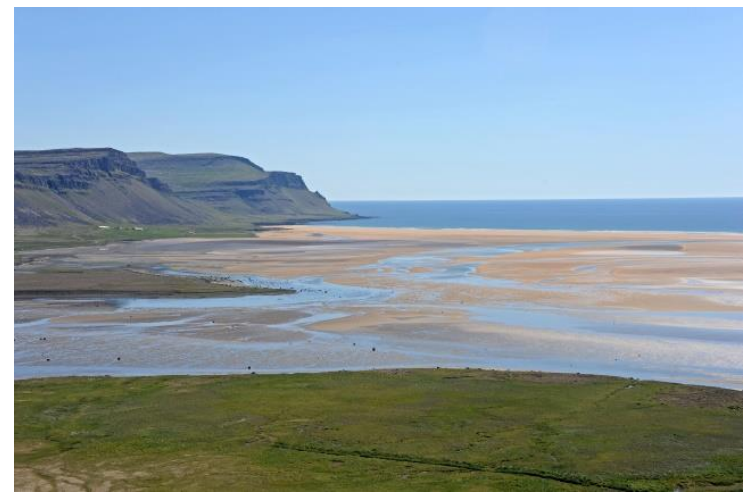

Photo 15 : Exemple de fjord ensablé par des sables rouges (oxydes métalliques) au débouché du fjord de Raudisandur (NW islandais). La faible profondeur et la largeur de la vallée glaciaire expliquent que la remontée de la mer ait pu former une barre littorale de $12 \mathrm{~km}$ de long (ici ancrage sud). (cliché. J-N Salomon).

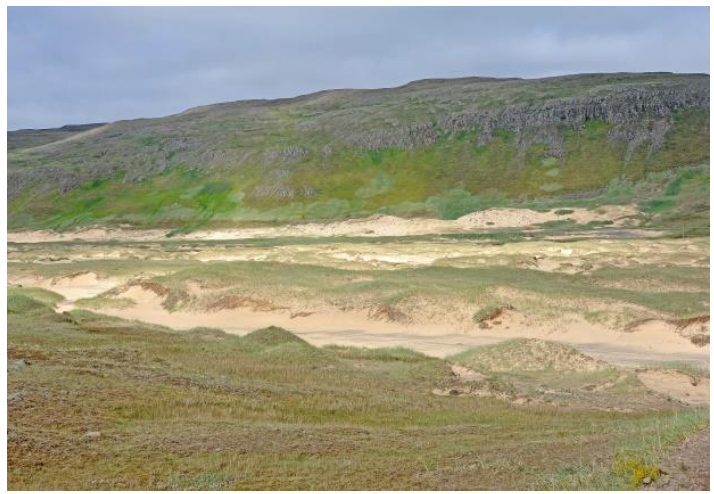

Photo 16 : Champ dunaire de Bredavik (NW de l'Islande). Les sables proviennent du broyage des moraines de fond et du lit du glacier, puis de leur remodelage par les actions marines qui y ont incorporé des tests de coquillages, enfin du vent qui a ramené les sables sur terre. Les écoulements intermittents ont ensuite tracé des lits. Il s'agit de sables très grossiers. (cliché. J-N Salomon).

\section{Comment expliquer ces différences drastiques?}

- La première cause semble être que l'inlandsis islandais a émis d'énormes émissaires au départ particulièrement larges (dépassant parfois $20 \mathrm{~km}$ ), qui se sont se rétrécis (à moins de $10 \mathrm{~km}$ ) à mesure du réchauffement de l'inlandsis, puis de la calotte glaciaire. Cette largeur a étendu l'action de creusement beaucoup plus latéralement qu'en profondeur, tout en produisant d'énormes quantités de débris. L'essentiel de la déglaciation a commencé vers - 14000 ans (période du Bölling) et s'est achevée à la fin du Dryas (vers - 10000 ans ; Van Vliet Lanoe et al., 2010), mais, avec quelques fluctuations, les fontes ont été continuelles. Elles se poursuivent de nos jours avec le réchauffement climatique. Les glaces ont eu largement le temps de modeler les vallées actuelles (et les fjords) dans leurs 
parties aval. Mais l'érosion par les glaciers a été différente de celle qui s'est produite en Norvège. En Islande, à l'origine les glaciers n'étaient pas contraints ou très peu. Ils ont donc creusé essentiellement latéralement (Photo. 2), puis à mesure de la fonte, ils se sont retrouvés resserrés entres des parois et devenus contraints. Ils ont alors creusé davantage en profondeur (les cannelures l'emportant sur les stries), mais peu de temps. La multiplication des chutes d'eau, spectaculaires (Photo. 17), plaide en faveur de la jeunesse du creusement. Aujourd'hui, la rareté des cours d'eau supra glaciaires montre que l'essentiel de l'écoulement est intra-glaciaire. Du coup, en surface, toute une série de morphologies se développent qui captent les eaux d'ablation (fissures, entonnoirs, puits, etc.) et de grands système de grottes (thermokarst). Les eaux ressortent donc par de grosses rivières à la sortie des conduits, mais de façon intermittente. Et de nombreux réservoirs internes au glacier peuvent céder d'un coup provoquant des crues brutales (jökulhlaup) qui modifient rapidement les morphologies.

- Ensuite, on observe une dissymétrie climatique qui s'est inscrite dans le paysage géomorphologique. Le Sud est très arrosé (Kirkjubaejarklaustur, $\mathrm{P}=1650 \mathrm{~mm}$ et Vestmannaeyjar, $\mathrm{P}=1600 \mathrm{~mm}$ ) avec des crues fluvio-glaciaires et des débâcles de plusieurs millions de $\mathrm{m}^{3}$. Le Nord est beaucoup plus sec, à tendance périglaciaire $(\mathrm{P}=650 \mathrm{~mm}$ à Egilsstadir, $\mathrm{P}=482 \mathrm{~mm}$ à Akureyri, et $650 \mathrm{~mm}$ au Laugafell mais plus en altitude (BOUT et al.,1955. Autrefois l'épaisseur des glaces devait y être bien inférieure à celle du Sud : partout, tant au Nord qu'au Nord-Est, on constate une forte production de débris de versants basaltiques (clapiers, coulées de blocs, solifluxion), la présence de sols polygonaux, de glaciers rocheux (ex: $20 \mathrm{~km}$ à l'est de Holar ; $10 \mathrm{~km}$ au nord-est de Hofsos, etc.) (Gudmunson, 2010), ou encore de corps fossiles devenus inactifs, la glace ayant fondu. Sur le littoral se pose alors la question des parts respectives du remaniement par la mer et/ou du soulèvement isostasique.

- L'Islande se situe sur la dorsale médio-atlantique si bien que les mouvements tectoniques notamment verticaux y sont anciens et importants. Par ailleurs, la surcharge glaciaire a entraîné une subsidence, puis la déglaciation un mouvement inverse (surrection), mais plus lent (Stewart et al., 2000), mettant en jeu l'élasticité de l'asthénosphère. Les fjells (plateau rocheux usés par le glacier) sont parsemés de placages morainiques (véritables tills) plus ou moins remaniés. Dès lors, quelles sont les parts respectives des contributions tectoniques et isostatiques ? La question est complexe car lors d'une déglaciation les glaciers émissaires commencent à fondre depuis la côte et logiquement le relèvement doit être plus rapide en zone littorale ce qui favorise la sédimentation. 
Enfin, il reste l'action de la mer. Plusieurs niveaux de plages et de terrasses marines, soulevées jusqu'à plus de 40 m, s'observent un peu partout (Einarson, 1961 ; Bodere, 1977). Les dépôts coquilliers marins observés au nord-est d'Husavik soulignent le soulèvement tectonique. Les plateformes littorales (strandflats) larges parfois de plusieurs centaines de mètres se remarquent tant au Nord comme autour du fjord Kalfalon (dont le glacier est issu de la calotte du Drangajökull), qu'au NordEst. Surtout, dans les vallées, les étalements de sédiments redistribués par la dérive littorale et les marées a engendré de barres, voire des îles et des cordons littoraux (Photo. 18) qui à leur tour génèrent, à l'amont des fjords, des chenaux anastomosés traversant des prairies, uniquement submergées lors des grandes crues. Seuls quelques gros blocs erratiques épars indiquent un dépôt glaciaire ancien. Ces morphologies indiquent que l'essentiel des dépôts a probablement été effectué lors d'un retrait rapide des glaciers émissaires, favorisé par le soulèvement isostatique consécutif $\mathrm{du}$ littoral. Puis la remontée du niveau de la mer, qui continue aujourd'hui, a joué son rôle ainsi que et la dérive littorale générant par exemple le tombolo double de Thordarhöfdi (au nord de Hofos).

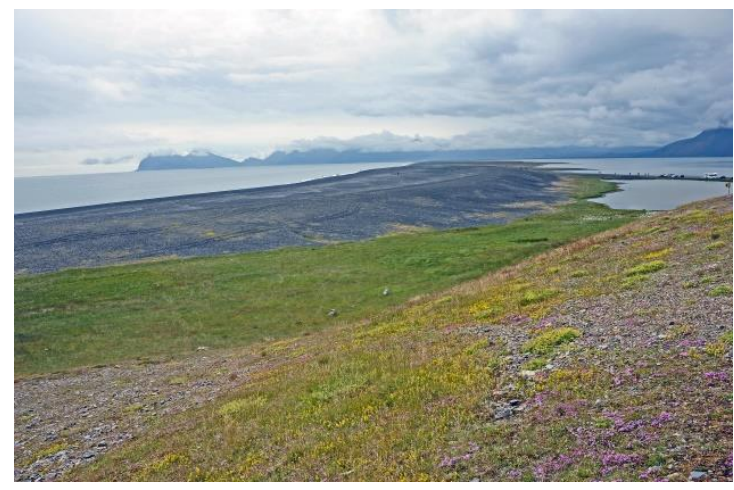

Photo 17 : Canyon de Jökulsárgljúfur et chutes de Hafragilfoss (Nord islandais). C'est le canyon le plus grand et le plus spectaculaire d'Islande. Il mesure environ $25 \mathrm{~km}$ de long pour $500 \mathrm{~m}$ de large et ses parois peuvent excéder les $100 \mathrm{~m}$. On pense que ce canyon a été créé lors d'une crue catastrophique de la rivière Jökulska après la dernière période glaciaire. Les chutes d'eau, nombreuses en Islande, sont le témoignage de la jeunesse du creusement, les cours d'eau étant loin de leur profil d'équilibre. (cliché. J-N Salomon).

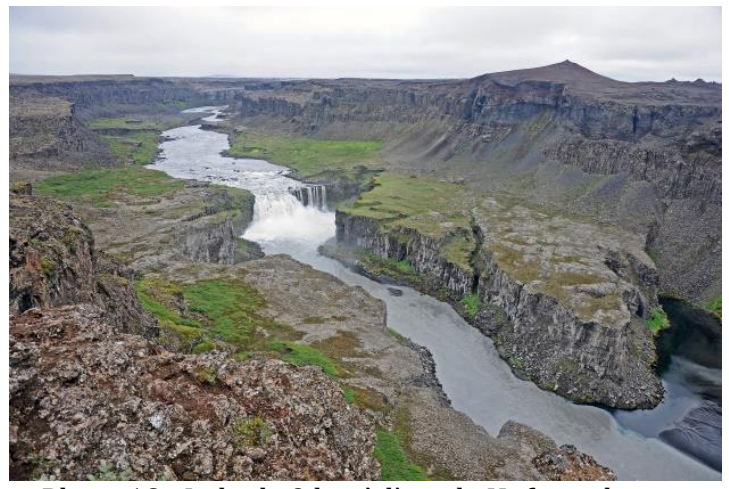

Photo 18 : Lido de $9 \mathrm{~km}$ à l'est de Hofn, isolant une vaste lagune. Le glacier émissaire du Vatnajökull le Svinafellsjokull ne parvient plus jusqu'à la mer, mais les torrents du front glaciaire amènent quantités de sédiments qui sont repris par les actions marines. La vallée glaciaire est très large. (cliché. J-N Salomon).

\section{Conclusion}

Les paysages morphologiques ont été décrits la plupart du temps à partir d'exemple types. La démarche est louable dans la mesure où elle permet de bien saisir un aspect paysager et l'essentiel de sa genèse. Mais c'est oublier qu'un paysage est la résultante 
de toute une histoire où de très nombreux paramètres sont intervenus (géologie, climat, topographie, érosion, accumulation, facteur temps, etc...). En fait seule une bonne connaissance des processus d'évolution permet d'appréhender la construction du paysage que l'on étudie et formuler des hypothèses crédibles. Ensuite, la méthode comparative trouve amplement sa justification. Ce que l'on a observé de visu à tel ou tel endroit de la planète doit servir de référence pour des analogies ou au contraire des divergences. Ensuite, bien entendu il convient de soumettre les scenarios avancés au contrôle des données scientifiques (microscopie, datations absolues, etc.). Pour en revenir aux fjords islandais, il est clair que leur genèse diffère de ce qui est généralement écrit dans les manuels.

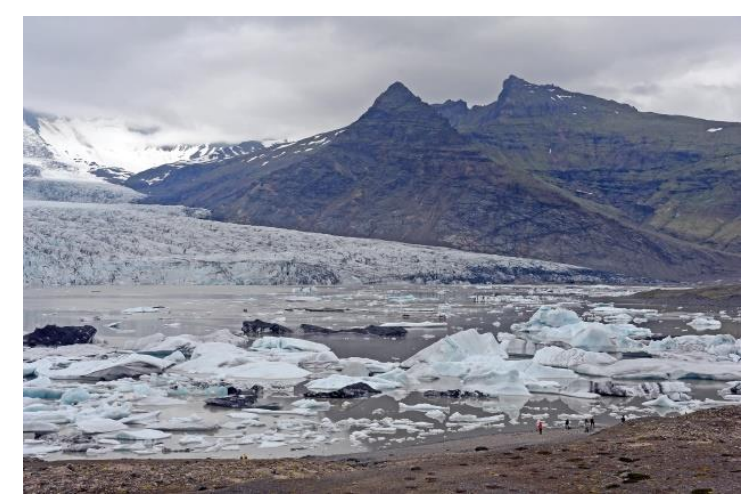

Photo 19 : Fjallsjökull - tourisme nature. Tout comme au Jökulsárlón (Sud du Vatnajökull) le spectacle du vêlage des blocs de glace sur canots pneumatiques, est très apprécié. (cliché. J-N Salomon).

En 2016, l'Islande a reçu 1,7 millions de visiteurs (pour un pays de 330000 habitants !). Le tourisme de nature est devenu la première ressource économique du pays ( $23 \%$ du PIB). La raison en est, certes, la désaffection pour les pays en conflit, mais aussi l'attractivité réelle, déjà ancienne, de ses sites naturels (glaciers, champs de lave, geysers, cascades, fjords, oiseaux, etc.). L'Islande se révèle surtout un terrain idéal pour l'appréciation de la nature (Photo. 19) l'apprentissage de la géomorphologie. Volcanisme, glaciaire, périglaciaire, hydrologie et étude littorale se comprennent vite, d'autant que l'absence ou la rareté de la végétation permet l'observation de façon claire.

\section{Références}

AhlmanN H.W. - 1954 - « Glacier variations and climatics' fluctuations » in American Geog. Soc. 51 p., 4 fig., 4 pl. photo. New-York.

AHLMANN H.W. ET THORARINSSONS S. - 1938 - «Vatnajökull : scientific results of the SwedishIceland investigations $1936-1937-1938$ », in Geogr. Annaler, pp. 171 - 233, Swedish Soc.for Anthr. and Geogr. Wiley Ed.

BODERE J.-C. - 1977 - «Les niveaux marins postglaciaires de l'Islande» in, Bull. Ass. Française Etude du Quaternaire, Vol. 14, № 4, pp. 76-78. 
BODERE J.-C. - 1985 - «La région côtière sud-est de l'Islande. Recherches géomorphologiques », Thèse d'État, 1826 p., 412 fig., 132 photos, 17 tab. Univ. Brest.

Bout P., Corbel J., Derruau M., Garavel L. Et PéGuy C.-P. - 1955 - « Géomorphologie et Glaciologie en Islande centrale », in Norois, Vol. 8, N 1, pp. 461-574.

DerRuAu M. - 1965 - «Précis de Géomorphologie », 415 pages. 162 figures, 61 planches hors texte. Masson Ed., 4 ème édition (nombreuses autres rééditions).

EINARSSON TR. - 1961 - « Das Meeresniveau an den Küsten Islands in postglazialer Zeit », in Neues Jb., Geol, Paläontol., 9, pp. 449-473. Ed. Schweizerbart, Stuttgart.

GUDMUNSON A. - 2010 - «Rock glaciers and relict debris bodies in Central North Iceland. Iceland in the Central Northern Atlantic: hotspot, sea currents and climate change ", IUEM - CNRS Plouzané, France.

MARTONNE E. DE (1910 et 1911) - «L'érosion glaciaire et la formation des vallées alpines ». Annales de géographie, XIX, 289-317 et XX, pp. 1-29.

SALOMON J.-N. - 2008 - « Géomorphologie sous-marine et littorale », manuel, 387 p., 154 Fig., 387 p., 7 tab., 30 photos, Presses Universitaires de Bordeaux. Prix Roger COQUE (2009) de la Société de Géographie.

STEWART I.S., SAUBER J. ET ROSE J. - 2000 - « Glacio-seismotectonics : ice sheets, crustal deformation and seismicity », in Quat. Sci. Rev., n¹9, pp. 1367-1389.

VAn Vliet Lanoe B., Guillou H., GudmundSSON A. Et SCNEIDER J.-L. - 2010 - « Glaciers and sea ice extent in Iceland during the Quaternary », 5 p., 3 fig., in IUEM - CNRS-Univ. Brest, Plouzané, France.

ZRYD A. - 2001 - « Les Glaciers », 325 p., Ed. Pillet, Saint-Maurice (Suisse). 